\title{
TUBERCULOUS PERICARDIAL EFFUSION
}

\author{
BY \\ S. SUZMAN \\ From the Cardiographic Department, Guy's Hospital \\ Received June 22, 1942
}

Tuberculous pericardial effusion is not common; and so the case described is of interest on account of the patient's age, the frequency with which the pericardial effusion required tapping, the relative absence of symptoms during most of the illness, and the stages through which the case passed from gross effusion to relative dryness at autopsy with only about $2 \mathrm{oz}$. of fluid in a greatly thickened pericardium.

\section{DESCRIPTION OF CASE}

A sailor, aged 23, was admitted into a hospital on August 16, 1940, for epigastric pain and frequent vomiting, which had begun three days previously. There had been a similar attack three months previously. There was continued pyrexia up to 102 . The diagnosis was apparently difficult until he complained of some sub-sternal tightness a few days later, when attention was directed to his heart. This was found to be enlarged, 1 inch outside the nipple line, but it was only after an X-ray that the diagnosis of pericardial effusion was established. Before this, on account of dullness at the left base of the lung with diminished breath sounds, a pleural effusion was suspected, but the X-ray did not confirm this. The blood pressure was typically low, 100/70.

Repeated tappings were performed as under:

August 23, 300 c.c. ; August 27, 400 c.c.; August 30, 500 c.c.; the fluid each time being sterile and clear and straw-coloured; September 6, 300 c.c.; and September 13, 550 c.c., the fluid being deeply blood-stained on these two occasions. (For later tappings see below.)

On Sept. 16 he was admitted to the Southern Hospital, where he came under my observation. The heart was greatly enlarged, both to left and right. The heart sounds were faint and muffled. Continued pyrexia was still present, varying between 99 and 103 . Symptoms were absent, except for sub-sternal tightness which he seemed to experience whenever the effusion increased; this was relieved after tapping. Tachycardia was a constant feature throughout, the pulse rate being about 100 to 120 . The blood sedimentation rate was $78 \mathrm{~mm}$. after one hour. Blood culture was sterile. A Mantoux test $(1$ in 10,000) was negative. And in spite of a negative guinea-pig test on two occasions, the diagnosis of tuberculous pericardial disease was made.

September 24, aspiration of 520 c.c. of old blood-stained fluid. There was no change in his blood pressure, 105/80, before and after, or in the cardiogram which showed the characteristic $\mathrm{T}$ wave inversions.

November 6 , aspiration of 600 c.c. of fluid, less blood-stained. He had remained fairly comfortable and without sternal pain. On this occasion an air replacement was done, and an X-ray afterwards showed the parietal layer of the pericardium, which measured half an inch thick. The cardiac shadow was not diminished after the tapping. X-ray on December 10, showed the air replacement to be absorbed, and an increase in the size of the effusion. A course of sulphapyridine (17 g.) was given about this stage, and brought down the temperature for a time. Towards the end of December he developed a dry cough and the temperature rose again to 102. He began losing weight and he had night sweats.

December 28, aspiration of 600 c.c. of amber-coloured fluid ; air replacement of 100 c.c. Fine crepitations were now heard, and an additional diagnosis of miliary tuberculosis of the lungs was made. He gradually became worse about the middle of January 1941, and was mildly delusional at times. On January 19, 1941, he became drowsy; coma set in and he died the next day.

Post-mortem examination. Pleura: light fibrous adhesions, obliterating both pleuræ.

Heart: Great dilatation of the pericardial sac, which contained only $2 \mathrm{oz}$. of amber fluid, but was much thickened by granulomatous tissue. A layer of friable yellow fibrin, uniting the thickened layers of the pericardium, up to $1 \mathrm{~cm}$. thick; no loculations. Heart itself only slightly enlarged; valves normal. 


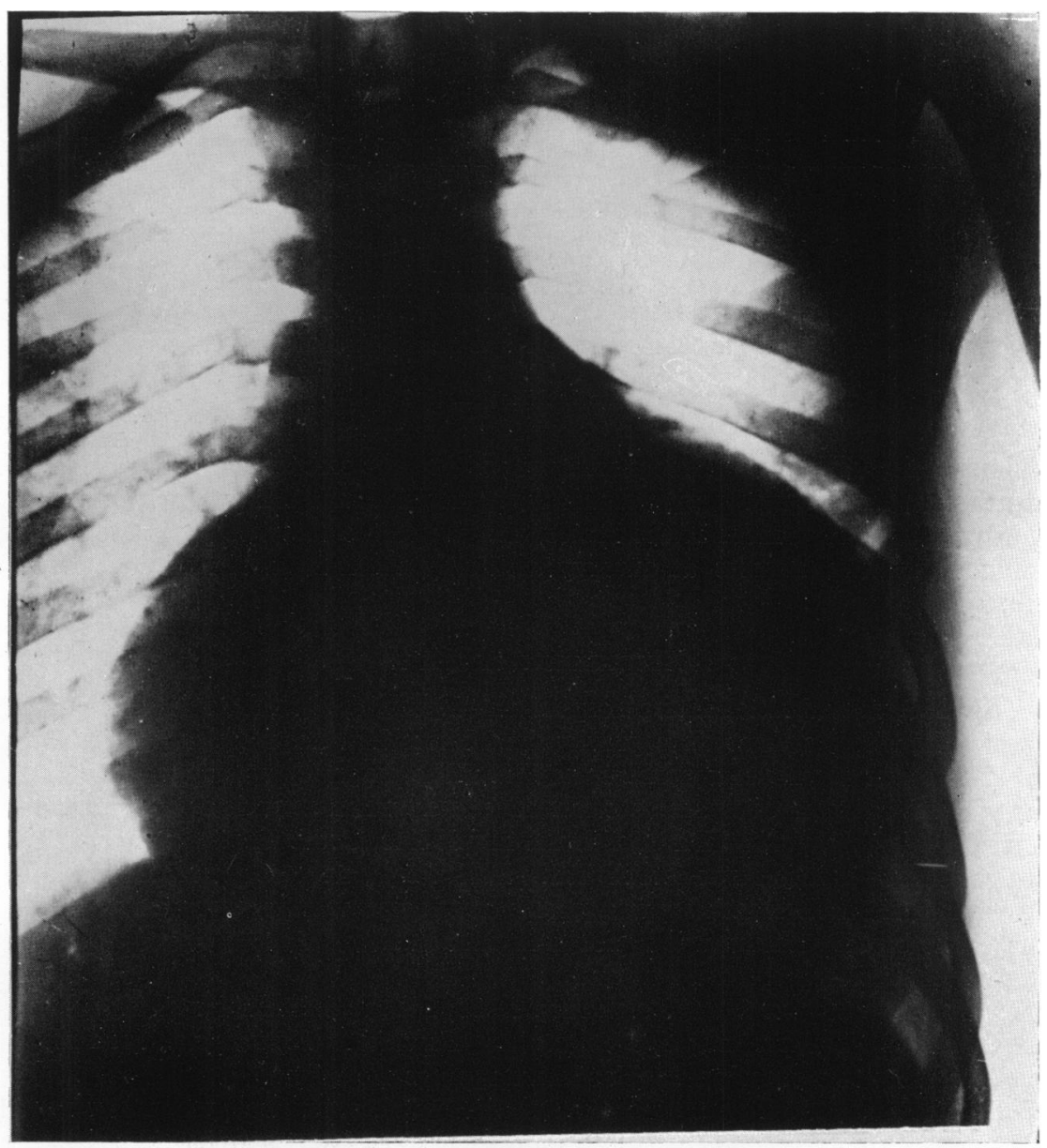

Lungs: Congested and closely studded with granulomatous miliary tubercles; no cavities. Caseous glands in both hila and at the bifurcation of the bronchi, two inches by one quarter inch in size on each side.

Liver: Cloudy swelling and a few miliary tubercles.

Spleen: Three times normal size; some tubercles.

Kidneys: Cloudy swelling with streaks of pus in the pyramids; studded with miliary tubercles.

Bladder: Infected with pus.

Prostate: Caseous tuberculosis present.

Brain: General œdema. Sero-meningitis over the base of the brain with a few scattered tubercles in the fissures. A tuberculoma, $0.5 \mathrm{~cm}$. in diameter, in the left occipital cortex.

Histological examination confirmed tuberculosis.

\section{Discussion of CASE}

There is no doubt that in this case the primary focus was a tuberculous gland in the mediastinum, secondarily affecting the pericardium. And later as the disease progressed, further dissemination occurred, causing generalised miliary tuberculosis. Another point of interest was the absence of lung symptoms until the final phase of his illness. Hæmorrhagic pericardial effusions are said to be characteristic of tuberculosis. I think this is an open question, and the probability of trauma when needling must be taken into full account. In this case the first three tappings were not blood-stained. The next two were deeply blood-stained, and the succeeding ones clearly pointed to the presence of old blood in the sac. The last specimen of pericardial fluid was only amber-coloured.

Another point of interest is that the cardiac shadow did not diminish after paracentesis, owing to the very thick and rigid pericardium. 


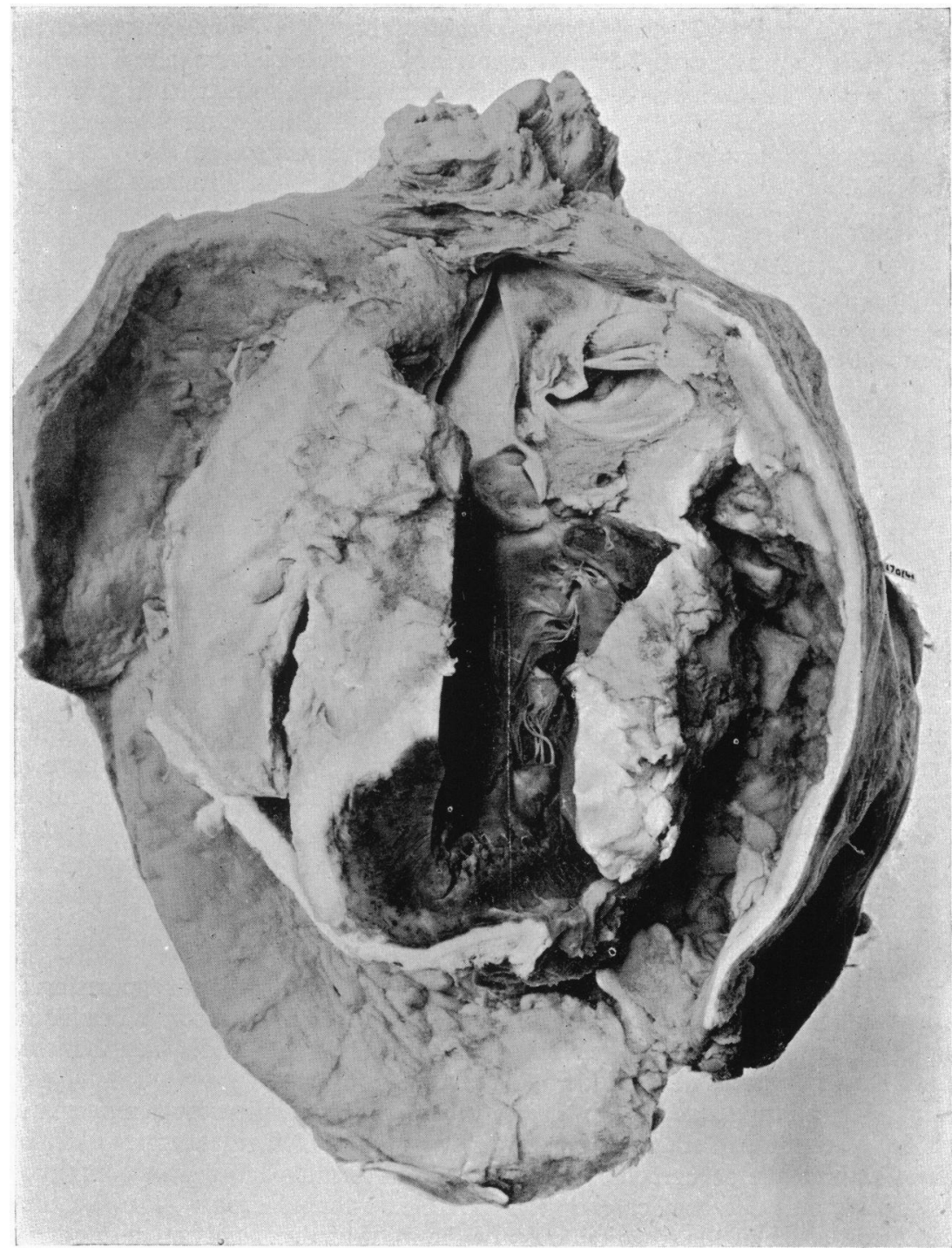

FIG. 2.-Photograph of the heart showing much thickening of the pericardium.

The negative results of laboratory findings, such as the absence of tubercle bacilli in the fluids and the failure of inoculation of guinea pigs to produce any result, should also be stressed.

\section{Post-Mortem Statistics at Guy's Hospital}

To assess the incidence of tuberculous pericarditis I have analysed 1893 autopsies at Guy's Hospital from January 1935 to June 1939. There were 102 tuberculous cases with lung involvement. There were 6 cases of tuberculous pericarditis, confirmed by histological section; 2 others with pericarditis were most probably tuberculous, judging from the post-mortem description, but cannot be included as there was no mention of tuberculosis macroscopically or microscopically. There was also one case of myocardial tubercle alone, without pericardial involvement. This makes an incidence of 0.3 per cent of all the cases that came to autopsy during this period, and 6 per cent of all cases that had tuberculous involvement in any organ. If the two probable cases were included it would raise the incidence among general cases only to slightly over 0.4 per cent, but it would raise the incidence among tuberculous cases to as high as 8 per cent.

As a comparison for incidence, Kornblum, Bellet, and Ostrum (1933) give figures of 1 per cent among general cases and 4 per cent among tuberculous cases (Philadelphia Hospital) : they reported 17 cases during a period of two years. The incidence of tuberculous pericarditis among non- 
Europeans in South Africa appears to be much greater than in the Guy's Hospital series; Heimann and Binder (1940) reported on 31 cases over a period of 14 years, which gives an average of over 3 cases a year. This contrasts with 1.3 cases a year at Guy's Hospital, and 8.5 cases a year at the Philadelphia Hospital. The number of autopsies is not mentioned in either instance.

Of the 6 cases, only one was diagnosed as such during life, the remainder being post-mortem findings. (The case described in this paper does not belong to this series.) In the remaining 5 the tuberculous pericarditis was a complication of some other disease. In all instances except in Case 3, the mediastinal glands were tuberculous.

Case 1. Male, aged 43. Myelogenous leukæmia. Post-mortem confirmation. Clusters of large yellow tubercles radiating outward to the hilar lymph glands which were caseous. Caseous nodules in lungs. Visceral and parietal layers of the pericardium covered by fibrinous exudate and studded with tubercles. Sac containing 300 c.c. of clear yellow fluid.

Case 2. Male, aged 70. Died on arrival at hospital from hæmorrhage from the lungs. No other clinical history.

Post-mortem: Lungs adherent to pleura. Surface extension of miliary follicles in the sub-pleural lymphatics. A reactivated Ghon focus in the left lower lobe. Mediastinal glands tuberculous. Pericardial sac thickened by recent lymphatic extension of the miliary type from the adjacent pleura. Light fibrous adhesions between both layers of the pericardium; no exudate.

Case 3. Male, aged 56. Syphilitic aortic incompetence with pleural effusion. Died two months after admission from cardiac failure.

Post-mortem: In addition to the widened syphilitic aorta, a left-sided pleural effusion of clear fluid. Two loculated empyemas in the right pleural cavity, their walls consisting of tuberculous granulation tissue. External surface of the pericardium covered with a delicate fibrinous exudate. Both layers moderately adherent and covered by a thick yellow granular fibrino-purulent exudate. No evidence of any tuberculous lesion in the lungs. Mediastinal glands likewise not affected by tubercle.

Case 4. Female, aged 4 months. Admitted for meningitis.

Post-mortem: Miliary tuberculosis of all the organs. Mediastinal glands caseous, with a caseous focus in the right upper lobe of the lung. Pericardium, miliary tuberculosis; no effusion.

Case 5. Male, aged 23. In hospital for over two months, and treated for increasing cough, pain in the chest, and later for pleural effusion. Sputum always free from tubercle bacilli. Pericarditis one month before death. The patient gradually went downhill and died. Ten months previously he had begun to have symptoms of lassitude and anorexia.

Post-mortem: Right lung studded with miliary tubercles. A fibro-caseous empyema, only 2 or 3 weeks old, in the lower third of the right pleural cavity. On the left side a much older thick walled empyema. Mediastinal glands caseous. Pericardium universally adherent owing to tuberculous pericarditis of considerable duration, caseating freely. Heart not enlarged. A large caseating nodule attached to the free border of the marginal cusp of the mitral valve. Section showed that the pericarditis was of many weeks' duration.

Case 6. Male, aged 14. Admitted three months before death on account of cough, dyspnœa, cyanosis, and pleural and pericardial effusions. Both cavities tapped once; clear fluid drawn off containing many lymphocytes. Tuberculous pericardial effusion diagnosed clinically, and injection into a guinea pig confirmed this by producing a tuberculous lesion. Later on, headaches and meningitic symptoms, followed quickly by the patient's death.

Post-mortem: Bilateral pleural effusions containing clear fluid. Old and recent pleurisy on both sides. A great deal of sub-pleural miliary tubercle. No evidence of any tuberculous lesions in the lung substance. Mediastinal glands caseous. Pericardial sac enormously thickened, only slightly adherent to the lungs, and containing much granulation tissue; easily broken down adhesions between the two layers of the pericardium. Heart of very small size. Section showed that the disease process was of considerable age. This was the only one of the six cases that was diagnosed as having tuberculous pericarditis during life.

\section{Discussion of Autopsies}

An interesting fact brought out by the analysis of the 1893 cases was that of the 102 with tuberculosis there were 27 with an excess of pericardial fluid alone, without any macroscopic evidence of pericardial disease; the amounts varied from slight excess to 200 c.c. of fluid. I feel that if sections were examined in these cases there would be microscopic evidence of miliary tubercle of the peri- 
cardium. Obviously this point is of academic rather than of practical interest as all these 27 cases died from their primary tuberculous lesions. Thus 26 per cent of the tuberculous cases had some excess of pericardial fluid. A comparison with 202 consecutive autopsies was made as to the frequency of excess pericardial fluid in 41 primary cardiac cases; 13 had excess of fluid (32 per cent). There were also 13 tuberculous cases in this small series, 3 of which had excess of fluid, the proportion happening to agree well with the 1893 cases which were analysed.

Of these 41 cardiac cases, 10 had rheumatic valvular disease and 12 had high blood pressure. 5 of the 10 in the rheumatic group ( 50 per cent) had excess pericardial fluid and 4 of the 12 ( 33 per cent) in the hypertensive group had excess fluid.

In the 1893 autopsies, only 14 cases of hæmopericardium occurred, and of these 14 cases only 1 was of tuberculous pathology. In this one the epididymis, prostrate, meninges, and lungs were affected; there was nearly $4 \mathrm{oz}$. of bloody fluid in the sac; the pericardium was normal to naked eye, but it was not sectioned. The remainder consisted of 8 cases of rupture of the myocardium following cardiac infarction; 1 case of rupture of a dissecting aortic aneurysm, 3 cases of carcinomatosis, and $\mathbf{i}$ case of high blood pressure with a cerebral hæmorrhage.

\section{SUMMARY}

A case of tuberculous pericarditis is described. It is suggested that in any obscure case of pericardial effusion, especially where this is large, tuberculosis should be thought of as a possible diagnosis; and that this is still more likely if tapping has to be repeated.

Among 1893 consecutive autopsies there were 6 cases of tuberculous pericarditis, but only one of these had been diagnosed clinically. Hæmopericardium did not occur in any of these cases. Some excess of pericardial fluid was found in about a quarter of all the tuberculous cases.

I would like to thank Dr. Willis for his kindness in performing some of the pericardial tappings, and Dr. Alan Daly for his permission to publish this case.

\section{REFERENCES}

Hannesson, H. (1941). Tubercle, 22, 79.

Heimann, H. L. and Binder, S. (1940). Brit. Heart J., 11, 165.

Kornblum, K., Bellet, S., and Ostrum, T. M. (1933). Amer. J. Rantgen, 29, 203. 